\title{
Comparison of Three Measures to Promote National Fitness in China by Mathematical Modeling
}

\author{
Pan Tang, ${ }^{1}$ Daqing Xu, ${ }^{1}$ Qing Dai, ${ }^{1}$ and Tingting Huang ${ }^{2}$ \\ ${ }^{1}$ Department of Public Physical Education, Hefei University, Hefei 230601, China \\ ${ }^{2}$ School of Mathematical Sciences, Anhui University, Hefei 230601, China \\ Correspondence should be addressed to Pan Tang; pan@hfuu.edu.cn
}

Received 22 January 2014; Accepted 27 January 2014; Published 26 March 2014

Academic Editor: Weiming Wang

Copyright (c) 2014 Pan Tang et al. This is an open access article distributed under the Creative Commons Attribution License, which permits unrestricted use, distribution, and reproduction in any medium, provided the original work is properly cited.

In this paper we established a mathematical model for national fitness in China. Based on a questionnaire and data of the General Administration of Sport of China and the National Bureau of Statistics of China, the dynamics for three classes of people are expressed by a system of three-dimensional ordinary equations. Model parameters are estimated from the data. This study indicated that national fitness put out by the Chinese government is reasonable. By finding the key parameter, the best measure to promote national fitness is put forward. In order to increase the number of people who frequently participate in sport exercise in a short period of time, if only one measure can be chosen, guiding people who never take part in physical exercise will be the best measure.

\section{Introduction}

To promote the development of mass sports in the new era of reform and opening-up in China and to improve national physical quality, the State Council launched a national fitness program on June 20, 1995 [1]. Now national fitness has got remarkable achievement. The survey shows that the number of people who often participate in sports activities in 2004 is more than 5.7\% the number in 1997 and the country has $28.2 \%$ of population who often take part in physical exercise in 2007 [1].

To better implement the national fitness program, the government takes some measures mainly on three aspects. The first one is conducting propaganda work, to make people aware of the importance of sports fitness and the implementation of the national fitness program and to arouse enthusiasm of the masses to participate in fitness [1]. The second one is guidance, that is, generally establishing institutions responsible for mass sports management work, such as social sports guidance center, national fitness guidance center, and social sports instructors [1]. The purpose is to help guide people to exercise. The last one is improvement of sports facilities. China has built and renovated various sports stadiums and actively promoted open stadiums. These have greatly improved material conditions of national fitness [1].

In [1] General Administration of Sport of China (GASC) also pointed out that, although we have got many achievements and experience, the mass sports in our country's overall development level is not high and the proportion of the population participating in regular physical exercise is not high. We still face many challenges [1].

According to the report of GASC [2], population can be separated into three classes. The first one is people who never take part in physical exercise. We call it as never exercise for short. The second one is people who occasionally take part in physical exercise. We call it as occasionally exercise for short. This class of people does weekly exercise activities of 1 to 2 times. The third one is people who often take part in physical exercise. We call it as frequently exercise for short. This class of people does weekly exercise activities of not less than 3 times, each time not less than 30 minutes and the exercise intensity above the average of person. Of course, the social sports instructors are included in the third-class of people.

So far, China has carried out three investigations of the current situation of mass sports in 1997, 2001, and 2008. According to the three surveys and the data of National 
Bureau of Statistics of China [3], the proportion of the three classes of people is listed in Table 1. Based on these data, we can see that the proportion of the third-class of people is not high. In the national fitness program (2011-2015), Chinese government put forward the target that the proportion of the third-class of people should be more than 32\% [2]. It is a natural question whether the goal can be attained. This is the first problem that we want to prove.

In addition, all of these programs cost an enormous sum of money every year, for example, over one year a total investment of sports funds 191,450,000,000 RMB on construction of site [1]. It is natural to ask how we can economically and quickly increase the number of frequently exercise people. Based on the national fitness program [1], there are some measures including propagation, guidance, and sports facilities. There is no doubt that the three measures together can promote national fitness. However, in order to save manpower and wealth of the country, we want to know which measure should be the best one if only one measure can be chosen. This is the second problem that we want to prove.

Currently, although some literatures reported the importance of national fitness, they only state or compare the achievement after the implementation of the national fitness program $[4,5,8,9]$. There are few literatures discussing the best strategy to improve the national fitness program. There are not any mathematical models to study how to improve the national fitness program. In this paper, based on a questionnaire and the data of GASC and the National Bureau of Statistics of China, we established a mathematical model and incorporated three measures and three classes of parameters. By numerical simulation and analysis, the key parameters are found and used to develop the best measure. Hopefully, it could provide the theory reference for the mass sports policy basis.

The paper is organized as follows. In Section 2 we establish a mathematical model based on a questionnaire and the data of the General Administration of Sport of China and the National Bureau of Statistics of China. Section 3 is devoted to parameter estimation, numerical simulations, and analysis of the system. The goal is to find the key parameter. We end the paper with a brief discussion.

\section{Modeling}

Based on the surveys, the population can be separated into three classes $x, y$, and $z$ [4-6]. $x, y$, and $z$ present the number of individuals who never, occasionally, and frequently participate in physical exercise at time $t$, respectively. Each class can be transformed to other classes. In order to better understand how to do transformation between the three-class groups and in order to get real data, we conducted a questionnaire survey. According to the questionnaire, by contacting the people who occasionally participate in exercise, that is, $y$, there is $10.57 \%$ of the first-class of people $x$ who become the second-class of people $y$. By contacting the people who frequently participate

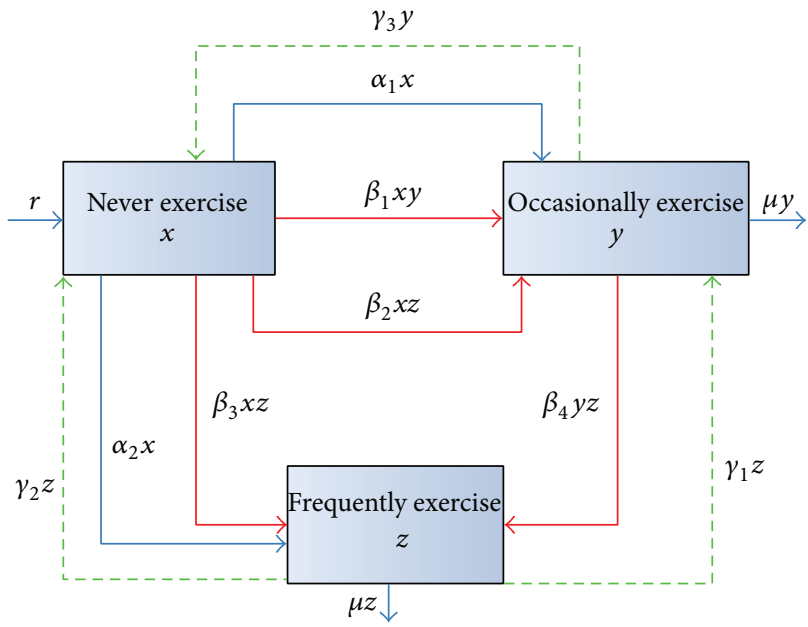

FIgURE 1: The diagram of class transformation. The red line represents transformation by contact, the blue line represents automatically transformation, and the green line represents reduction of the times of exercise.
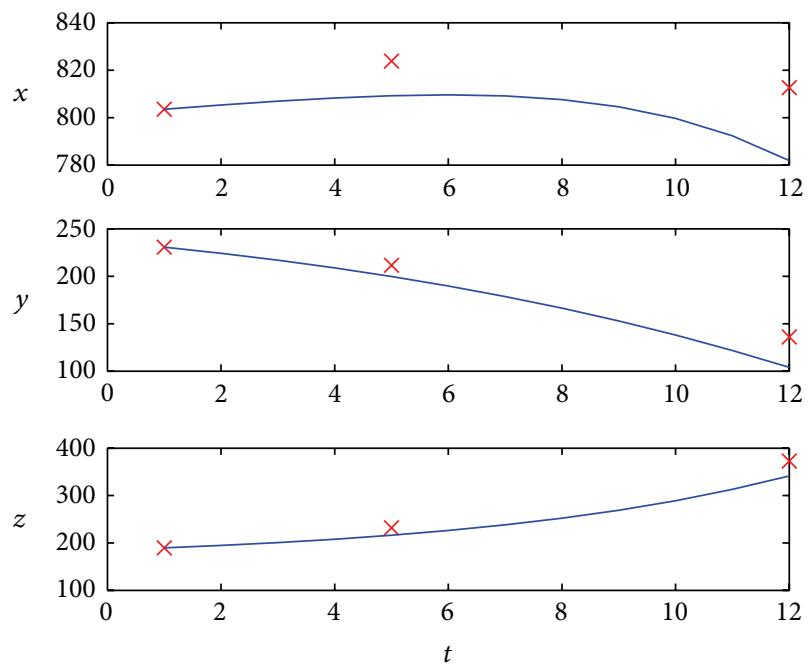

Figure 2: The fitting between the real number of three classes of people and the solution of the system. The red is real number and the blue line is the solution.

in exercise, that is, $z$, there are $8.89 \%$ and $11.2 \%$ of the firstclass of people $x$ who become the second-class of people $y$ and the third-class of people $z$, respectively; and there is also $17.6 \%$ of the second-class of people $y$ who become the thirdclass of people $z$. Because of propaganda, there are $26.03 \%$ and $21.2 \%$ of the first-class of people $x$ who automatically become the second-class of people $y$ and the third-class of people $z$, respectively. At the same time, some people reduced the times of exercise because of the lack of sport facilities. For the third-class of people $z$, there are $24.52 \%$ and $15.93 \%$ of the class of people who become the second-class of people $y$ and the first-class of people $x$, respectively. And there is $15.04 \%$ of the second-class of people $y$ who become the first-class of 
TABLE 1: Data of survey for the proportion of the three classes of people.

\begin{tabular}{lccccc}
\hline Year & First-class (\%) & Second-class (\%) & Third-class (\%) & $\begin{array}{c}\text { The total number of } \\
\text { populations (million) }\end{array}$ & References \\
\hline 1996 & 65.65 & 18.85 & 15.5 & 1223.89 & {$[3-5]$} \\
2000 & 65 & 16.7 & 18.3 & 1267.43 & {$[3-5]$} \\
2007 & 61.5 & 10.3 & 28.2 & 1321.29 & {$[3,6]$} \\
\hline
\end{tabular}

TABle 2: Parameters.

\begin{tabular}{ll}
\hline Parameters & Description \\
\hline$r$ & Intrinsic rate of increase of human \\
$\alpha_{1}$ & Carrying capacity of population \\
$\alpha_{2}$ & Automatic transformation rate of individual from the class $x$ to the class $y$ because of propaganda \\
$\beta_{1}$ & Automatic transformation rate of individual from the class $x$ to the class $z$ because of propaganda \\
$\beta_{2}$ & Transformation rate of individual from the class $x$ to the class $y$ by contacting individuals at the class $y$ \\
$\beta_{3}$ & Transformation rate of individual from the class $x$ to the class $y$ by contacting individuals at the class $z$ \\
$\beta_{4}$ & Transformation rate of individual from the class $x$ to the class $z$ by contacting individuals at the class $z$ \\
$\gamma_{1}$ & Transformation rate of individual from the class $y$ to the class $z$ by contacting individuals at the class $z$ \\
$\gamma_{2}$ & Automatic transformation rate of individual from the class $z$ to the class $y$ because of the lack of sports facilities \\
$\gamma_{3}$ & Automatic transformation rate of individual from the class $z$ to the class $x$ because of the lack of sports facilities \\
$\mu$ & Automatic transformation rate of individual from the class $y$ to the class $x$ because of the lack of sports facilities
\end{tabular}

TABLE 3: Birth rate and death rate.

\begin{tabular}{lcc}
\hline Year & Birth rate (per year) & Death rate (per year) \\
\hline 1996 & 0.01698 & 0.00656 \\
2000 & 0.01403 & 0.00645 \\
2007 & 0.0121 & 0.00693 \\
\hline Mean & 0.01437 & 0.00665 \\
\hline
\end{tabular}

TABLE 4: Value of parameters.

\begin{tabular}{lcc}
\hline Parameters & Values (per year) & Reference \\
\hline$r$ & 0.00772 & {$[3]$} \\
$K$ & $1.6 * 10^{9}$ & {$[7]$} \\
$\alpha$ & 0.02614 & Estimated \\
$\beta$ & 0.0089 & Estimated \\
$\gamma$ & 0.01512 & Estimated \\
$\mu$ & 0.00665 & {$[3]$} \\
\hline
\end{tabular}

people $x$. The diagram of class transformation is in Figure 1. Then we obtain the following system:

$$
\begin{aligned}
& \frac{d x}{d t}=r x\left(1-\frac{x}{K}\right)-\beta_{1} x y-\beta_{2} x z-\beta_{3} x z-\alpha_{1} x \\
& +\gamma_{2} z+\gamma_{3} y-\alpha_{2} x \\
& \frac{d y}{d t}=\beta_{1} x y+\beta_{2} x z+\alpha_{1} x+\gamma_{1} z-\gamma_{3} y-\beta_{4} y z-\mu y, \\
& \frac{d z}{d t}=-\gamma_{1} z-\gamma_{2} z+\beta_{3} x z+\beta_{4} y z+\alpha_{2} x-\mu z .
\end{aligned}
$$
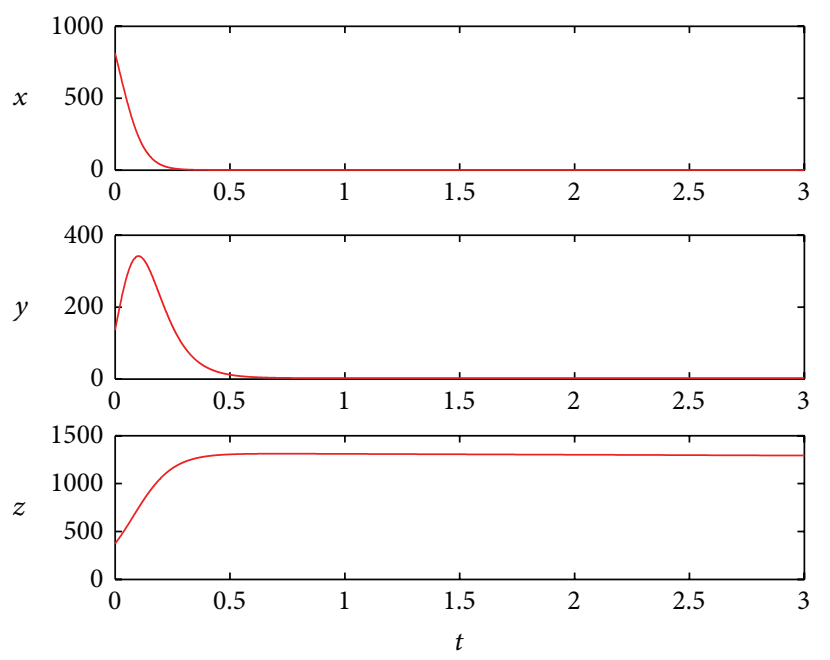

FIGURE 3: The system approaches a stable positive equilibrium state. The number of people who frequently participate in physical exercise is increased.

All the parameters are listed in Table 2. The aim is to study the impact of lead/guidance, conduct propaganda, and sports facilities on national fitness development. In detail, it is to study the effect of these parameters $\alpha, \beta$, and $\gamma$ on the dynamics of the national fitness model and to find the key parameter.

\section{Simulation and Analysis}

Since the system is very complex, theoretical analysis is very difficult and we use numerical simulation to analyze 


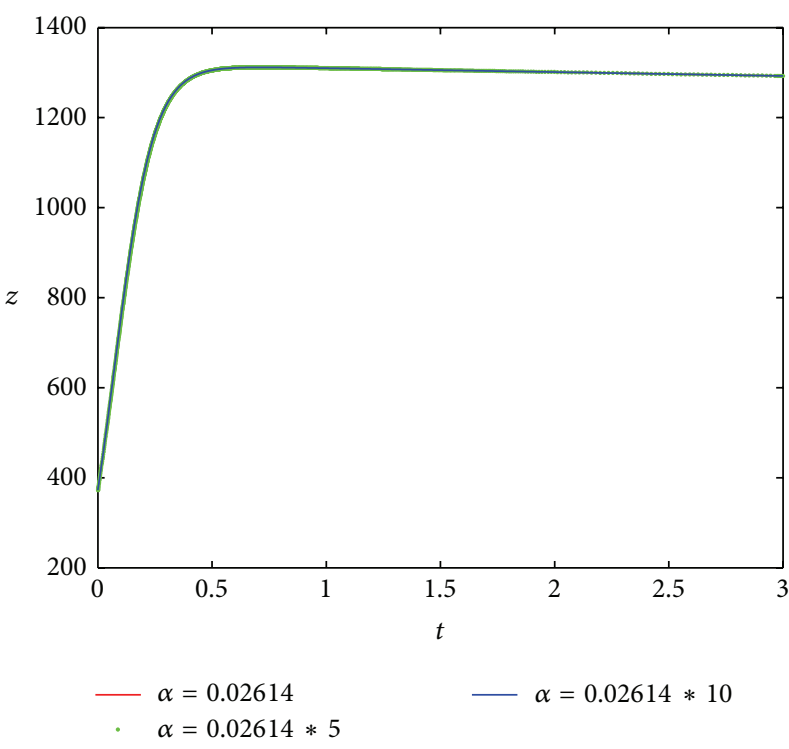

(a) Changing the value of $\alpha$

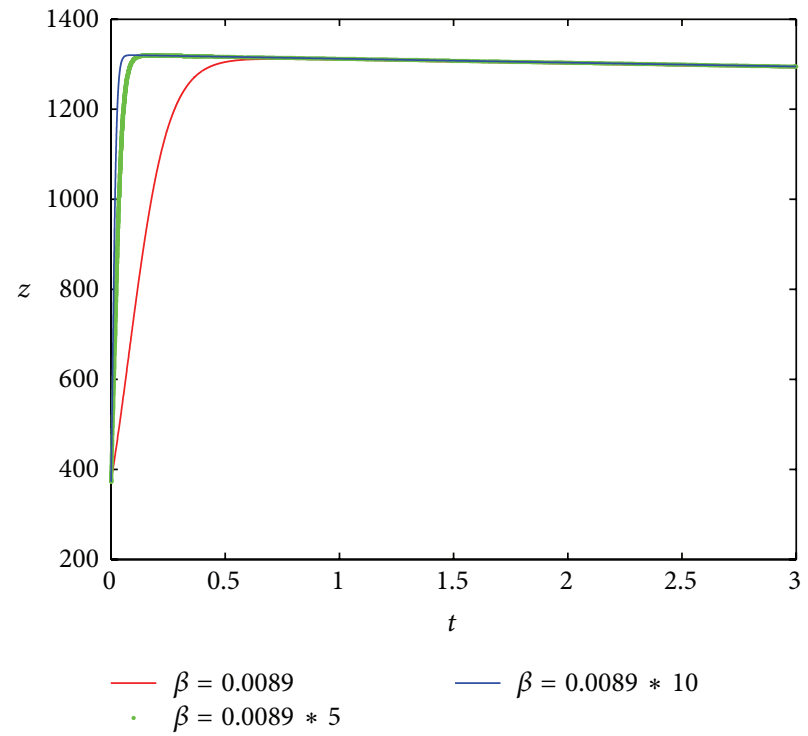

(b) Changing the value of $\beta$

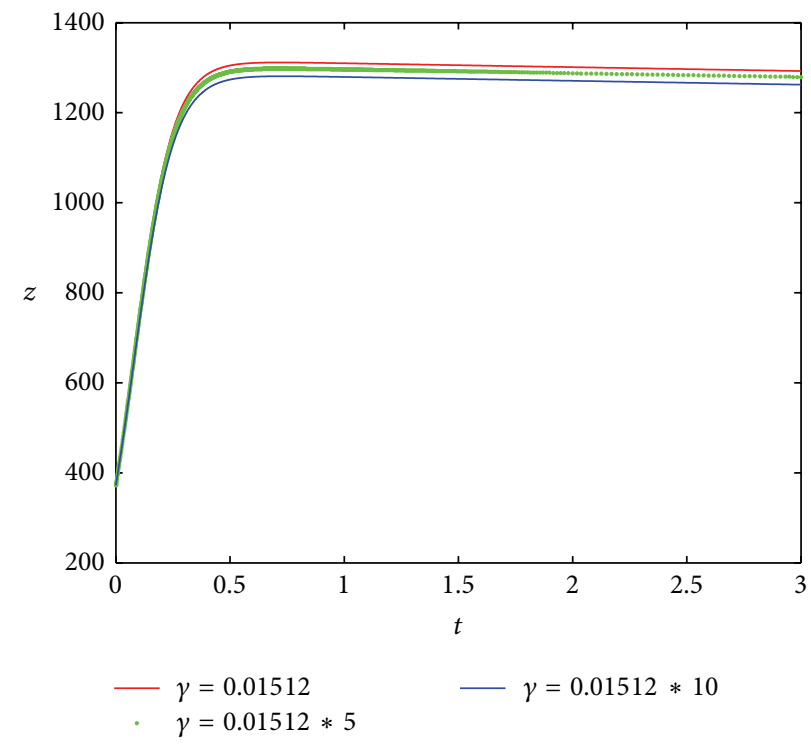

(c) Changing the value of $\gamma$

FIGURE 4: Increasing the value of $\alpha$ and $\beta$ and reducing the value of $\gamma$ can all increase the number of $z$. The impact of $\beta$ on the system is the biggest. The impact of $\gamma$ on the system is little bigger than the impact of $\alpha$.

the dynamical behaviors based on the real data. According to the data of the National Bureau of Statistics of China [3], the birth rate and the death rate of population in China are listed in the Table 3. In 1991, China Academy of Sciences published a report about the productivity and population carrying capacity of the land resource in China. The carrying capacity is 1600000000 [7]. To obtain the value of other parameters, we performed some estimations based on the data of GASC and the National Bureau of Statistics of China. For convenience, we assume that $\alpha_{1}=\alpha_{2}=\alpha, \beta_{1}=$ $\beta_{2}=\beta_{3}=\beta_{4}=\beta$, and $\gamma_{1}=\gamma_{2}=\gamma_{3}=\gamma$. Then, we estimate the three parameters based on the minimal error method and obtain the fitting figure (Figure 2). Finally, the value of all parameters is listed in Table 4. Based on these values of parameters, the system approaches a stable positive equilibrium state (Figure 3). From Figure 3, we can see that the number of people who frequently participate in exercise will increase. Hence, the goal of Chinese government in the national fitness program (2011-2015) can be attained.

Now we want to solve the second question. By changing the value of three parameters $\alpha, \beta$, and $\gamma$, we investigate the impact of these three measures on the number of three classes of people $x, y$, and $z$ (Figure 4). From Figure 4(a), we can see that $\alpha$ cannot infect the dynamics of the system. This means that intensifying propaganda is not obvious to increase the number of people who frequently participate in sport 


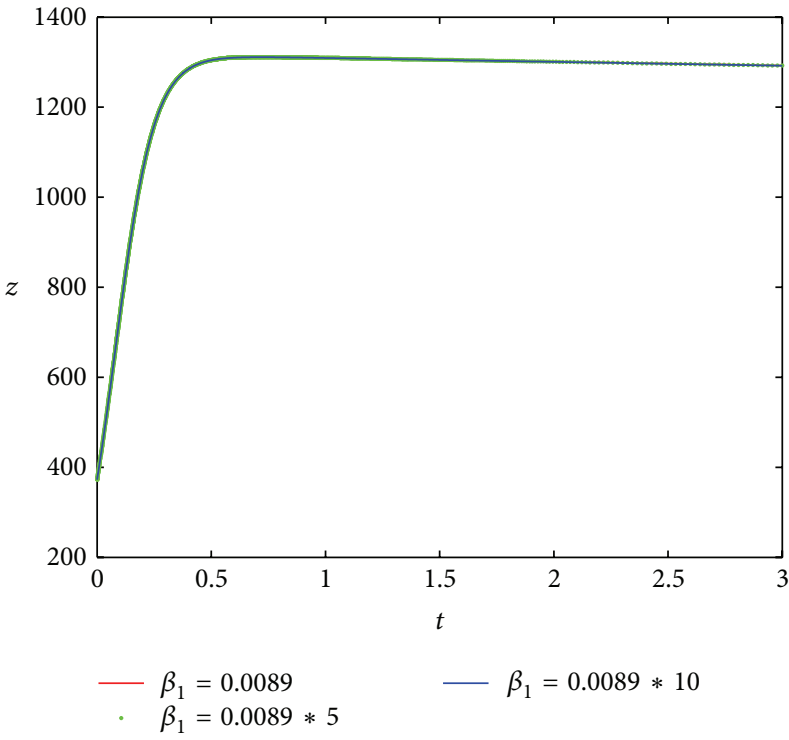

(a) Changing the value of $\beta_{1}$

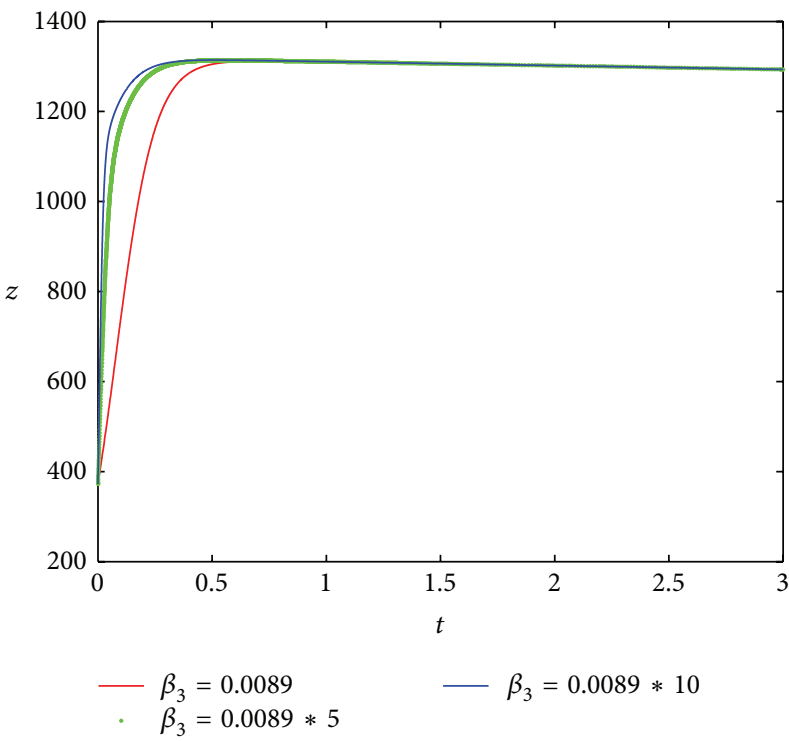

(c) Changing the value of $\beta_{3}$

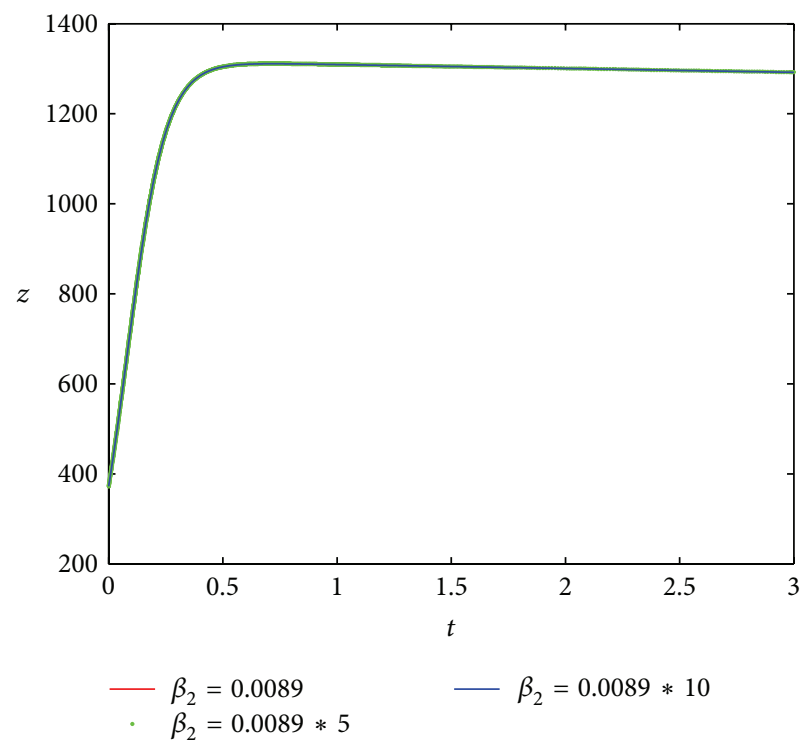

(b) Changing the value of $\beta_{2}$

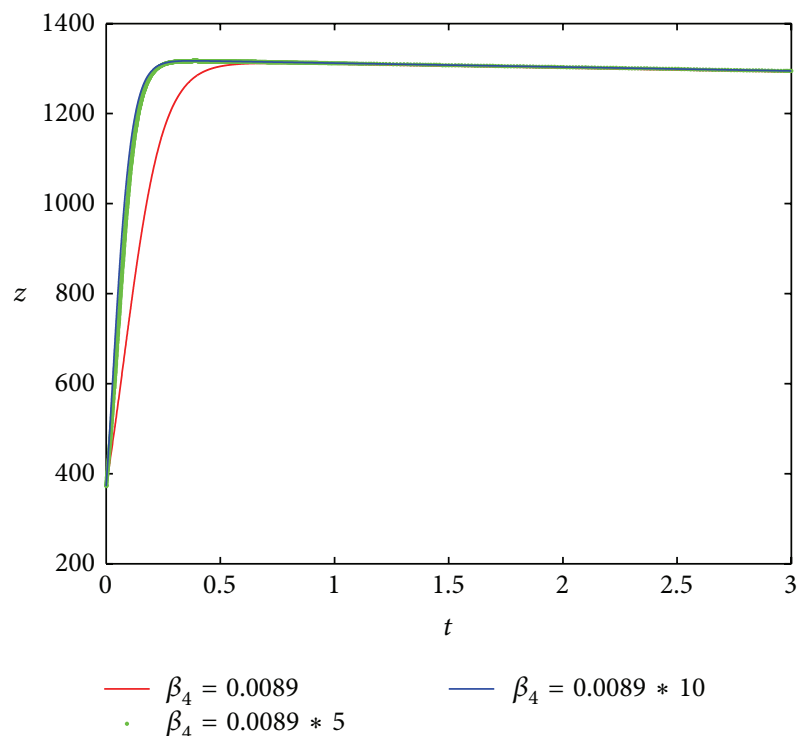

(d) Changing the value of $\beta_{4}$

Figure 5: The impact of four different $\beta$ on the system.

exercise. However, Figure 4(b) displays the fact that $\beta$ can strongly infect the dynamics of the system. In other words, by providing guidance for the first- and second-class of people, more and more people will frequently participate in sport exercises. At last, improving sport facility can increase the number of the people who frequently participate in sport exercise, which can be seen from Figure 4(c).

Now we want to know which class of people provided guidance that can lead to the best effect. By changing the four different parameters $\beta_{1}, \beta_{2}, \beta_{3}$, and $\beta_{4}$ in the original system (1), we find that $\beta_{3}$ and $\beta_{4}$ have stronger effect in the system (Figure 5). This means that only the third- class of people $z$ guiding the other classes of people $x$ and $y$ can lead to better effect.

Next, by changing the values of $\beta_{3}$ and $\beta_{4}$, we compare the effect of guiding between the first- and the second-class of people (Figure 6) and we can obtain the following results. First, from the black line we can see that guiding both the first- and second-class of people will increase the number of the third-class of people quickly, which means the effect by guiding the first two classes of people is the best one. Second, if we want to increase the number of people who frequently participate in sport exercise in a short period of time, guiding the first-class of people will be the best measure. Lastly, if we 


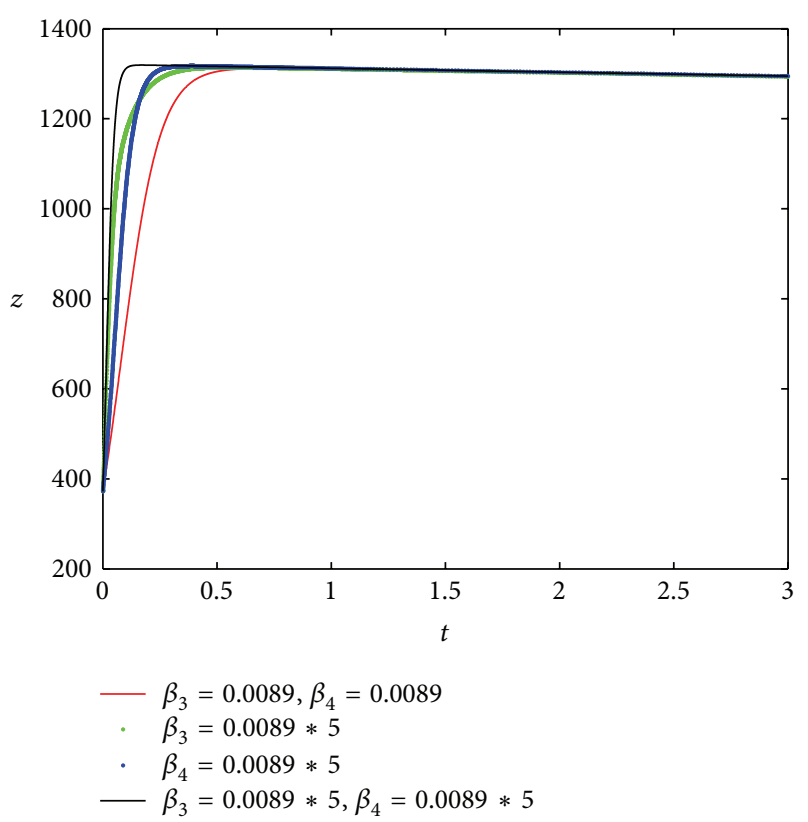

FIgURE 6: Comparing the effect of guiding for different classes of people.

want to increase the number of the third-class of people over a long period of time, guiding the second-class of people will be the best measure.

\section{Discussion}

As we know, national fitness can improve national physical quality. National fitness is promoted all over the world. Furthermore, Chinese government put forward the target that the proportion of the third-class of people should be more than $32 \%$ [2]. It is needed to consider whether the goal can be attained and how to better and faster achieve the anticipated goal. In previous literatures and reports, all conclusions are from reports or experiences. However, there are not any results based on mathematical theory. In this paper, based on a questionnaire and the data of the General Administration of Sport of China and the National Bureau of Statistics of China, we established a mathematical model for three classes of people and represented three measures by three classes of parameters. By numerical simulation and analysis, the key parameter $\beta$ is found. Furthermore, the two previous problems can be answered.

By simulation and analysis based on our mathematical model, the following conclusions can be obtained. First, the number of people who frequently participate in sport exercises will increase definitely and then the goal can be attained. Second, we found that conducting propaganda cannot increase obviously the number of people who frequently participate in sport exercises. In reality, people can be impressed by much propaganda. However, it is always difficult to act due to various reasons. Third, sports facilities are not so important as people imagine. From the figures we can see that the effect of reducing the value of $\gamma$ is not stronger.
In fact, there are many ways to participate in physical exercise without sports facilities.

Lastly, it is very important to provide guidance for all people.From the simulation of the model, we can see that the effect of parameter $\beta$ is the biggest. Furthermore, increasing the value of $\beta$ by five times can obtain better effect than increasing the value by ten times. Hence, proper guidance will enable more people to participate in physical exercise frequently. Currently, there are many ways to provide guidance, for example, establishing national fitness guidance center and providing social sports instructor. The report of the General Administration of Sport of China in 2009 [10] also fully affirmed that social sports instructors play a very important role in national fitness. In addition, by comparing the effect between $\beta_{3}$ and $\beta_{4}$, we found some different results. If we want to increase the number of people who frequently participate in sport exercise in a short period of time, guiding the first-class of people will be the best measure. If we want to increase the number of the third-class of people in a long period of time, guiding the second-class of people will be the best measure. Hence, we can provide more and more social sports instructors only for the first- and the second-class of people in the future.

In summary, these conclusions are also consistent with the actual situation. Furthermore, based on the mathematical modeling and the real data, we think that guidance is the best measure for national fitness. Hopefully, these conclusions could provide the theory reference for the mass sports policy basis.

\section{Conflict of Interests}

The authors declare that there is no conflict of interests regarding the publication of this paper.

\section{Acknowledgments}

This research is supported by Sports and Social Science Foundation of Sports Bureau of Anhui Province (ASS2012238). The authors would like to specially thank Professor Huaiping Zhu, York University of Canada, for his guidance and help. Also, they would like to thank anonymous reviewers for the very helpful suggestions which improved greatly this paper.

\section{References}

[1] General Administration of Sport of China, "Outline of the national fitness program (The implementation of fifteen years)," pp. 1-66, 2011.

[2] General Administration of Sport of China, "The national fitness program (2011-2015)," 2011, http://www.chinasfa. net/ArtDetail.aspx?aid=6537.

[3] National Bureau of Statistics of China, http://www.stats.gov.cn/.

[4] Z. J. Zhang and C. Liu, "Commentary on the development of Chinese sports population since reform and opening," Journal of Physical Education Institute of Shanxi Normal University, vol. 22, no. 1, pp. 19-22, 2007. 
[5] X. R. Zhou and M. Y. Tan, "Comparative research on our mass sports condition during two times investigation," Sport Science, vol. 24, no. 7, pp. 12-15, 2004.

[6] General Administration of Sport of China, "In 2007 China urban and rural residents to participate in physical exercise situation survey bulletin," 2012, http://www.gov.cn/ test/2012-04/19/content2117453.htm.

[7] China Academy of Sciences, "Population carrying capacity of China," 1991, http://zh.wikipedia.org.

[8] X. P. Lin, "The research on China mass sports developing tendency toward 2010," China Sport Science and Technology, vol. 37, no. 11, pp. 10-13, 2001.

[9] P. Tang, "Analysis of the characteristics of the national sports meeting," Sports Culture Guide, vol. 12, pp. 9-11, 2010.

[10] General Administration of Sport of China, "Active in the mass sports, social sports instructor was generally recognized," 2009, http://www.chinasfa.net/ArtDetail.aspx?aid=5306. 


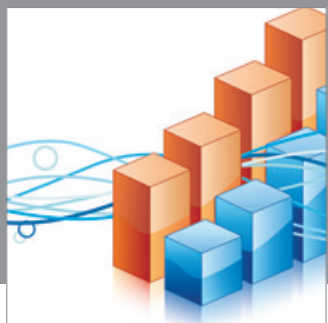

Advances in

Operations Research

mansans

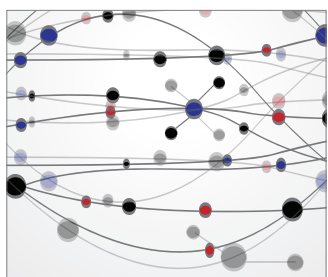

The Scientific World Journal
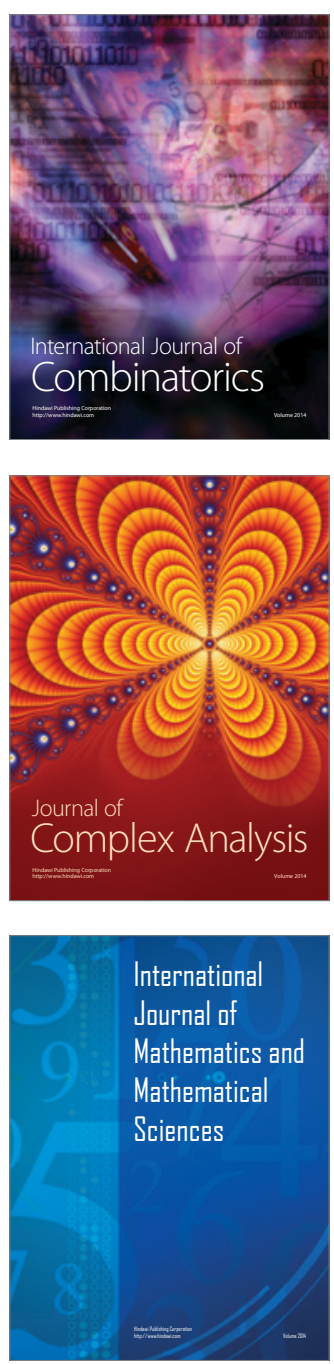
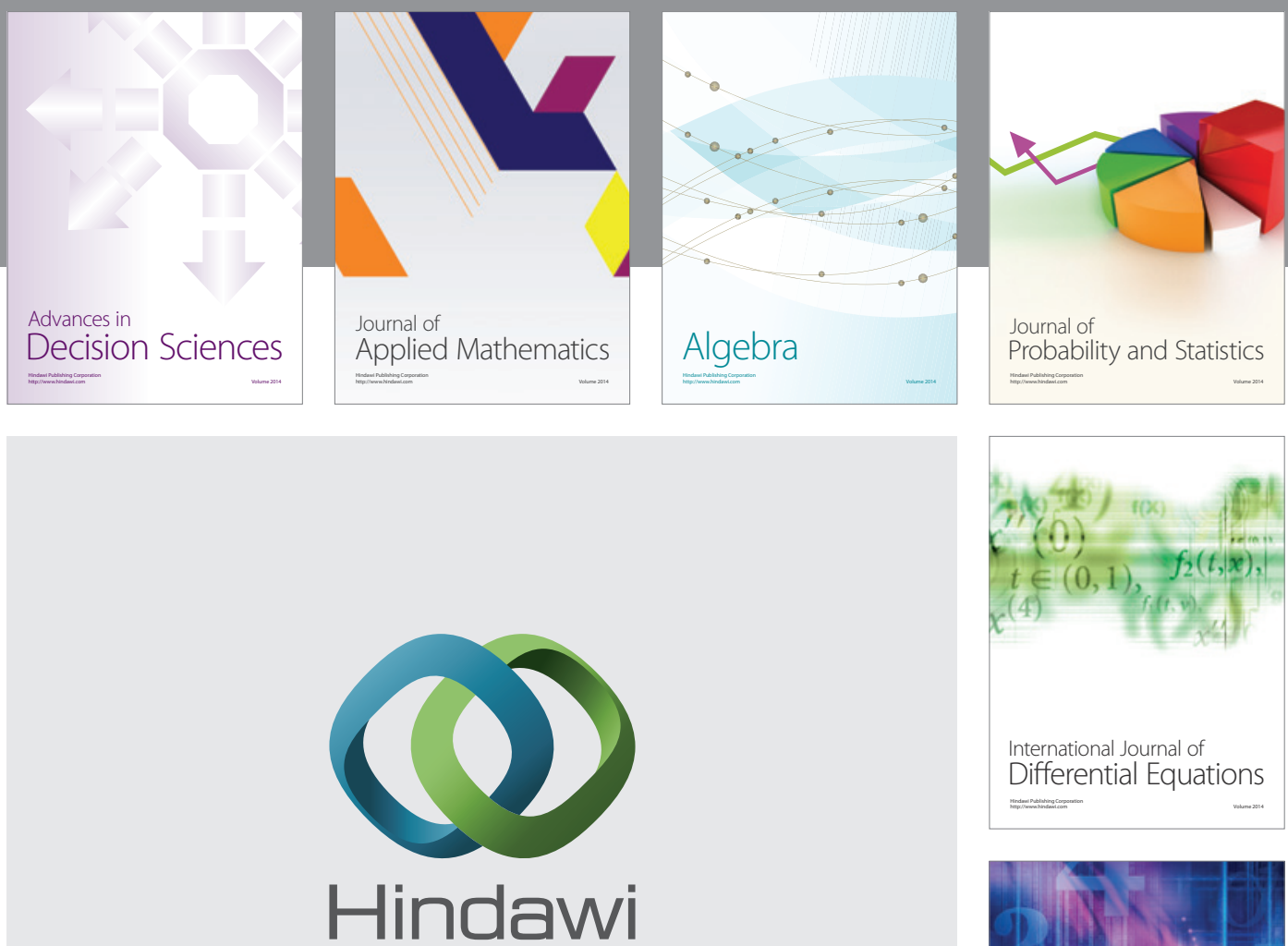

Submit your manuscripts at http://www.hindawi.com
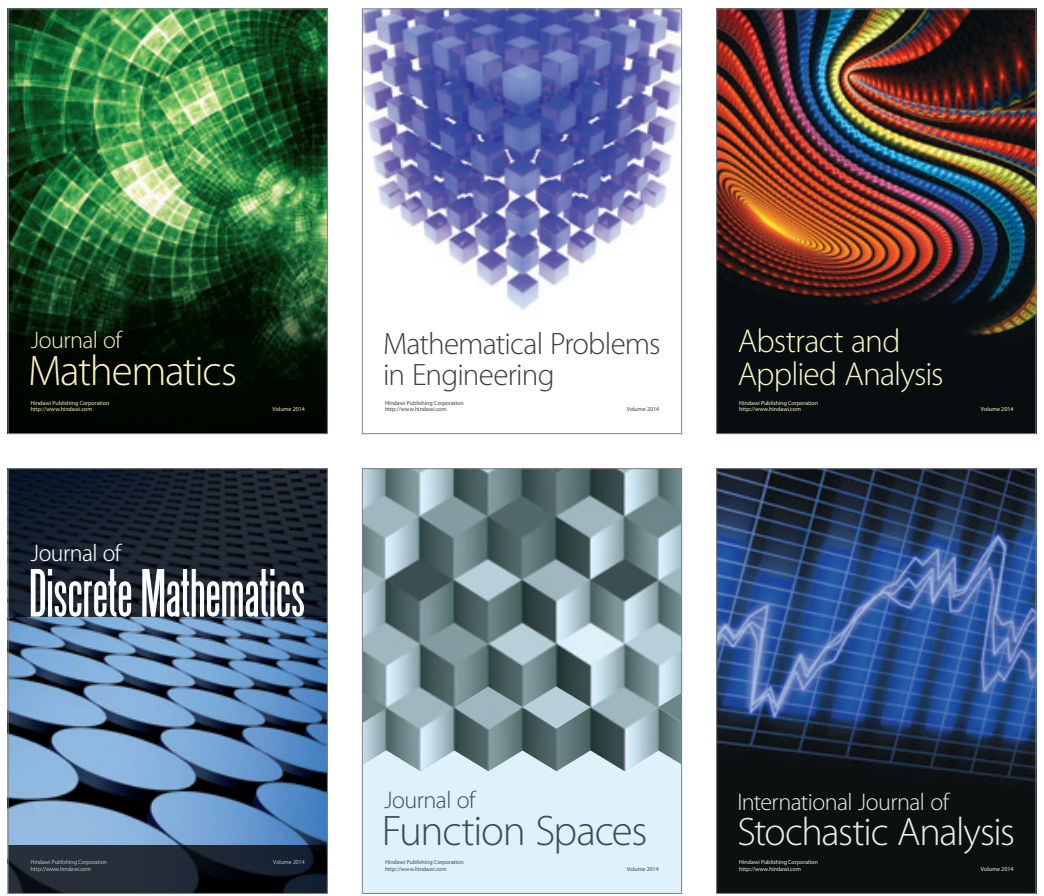

Journal of

Function Spaces

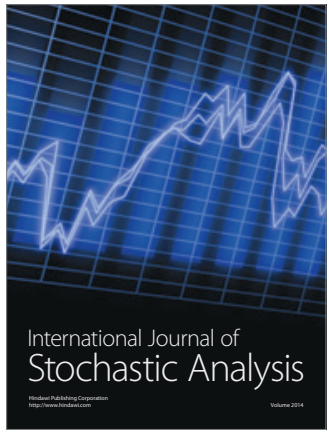

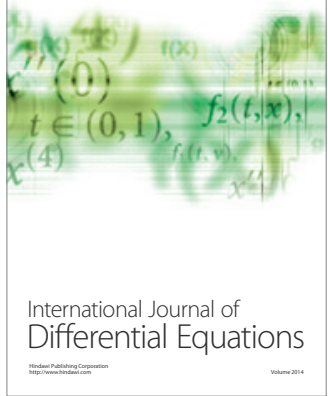
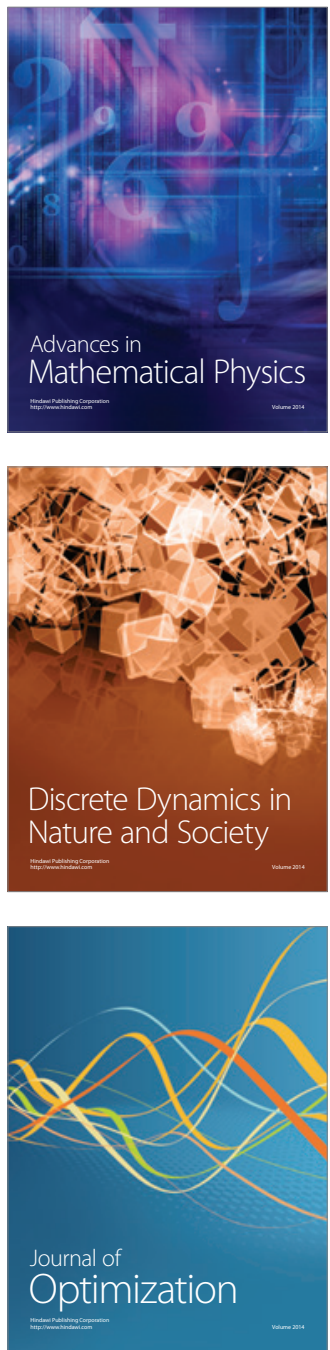